\title{
PUGH CONTROLLED CONVERGENCE AND SOCIAL CHOICE THEORY
}

\author{
Nicholson, John Morgan; Collopy, Paul \\ The University of Alabama in Huntsville
}

\begin{abstract}
The Pugh Method of Controlled Convergence is evaluated based on social choice theory, both from an axiomatic basis, and by examining all possible cases of attribute ranks for a range of numbers of alternatives and numbers of attributes. The evaluation shows that, for a typical Pugh application, concept selection varies with the arbitrary choice of datum or is simply incorrect in about one-third of the cases. While there are merits to the iteration steps and creation of new alternatives within the Pugh method, a simpler and more expressive concept ranking procedure can give far superior results.
\end{abstract}

Keywords: Collaborative design, Design theory, Design methods, Systems Engineering (SE)

Contact:

Collopy, Paul

The University of Alabama in Huntsville

Industrial and Systems Engineering and Engineering Management

United States of America

pdc0056@uah.edu

Cite this article: Nicholson, J.M., Collopy, P. (2019) 'Pugh Controlled Convergence and Social Choice Theory', in Proceedings of the 22nd International Conference on Engineering Design (ICED19), Delft, The Netherlands, 5-8 August 2019. DOI:10.1017/dsi.2019.130 


\section{INTRODUCTION}

Following the 2014 National Science Foundation's Science of Systems Engineering Workshop (Collopy and Mesmer, 2015), the authors have pursued a research agenda to place systems engineering on a more solid theoretical basis. The advantages of a theoretical foundation are many, but a key advantage is the ability to discriminate among methods, processes and tools so that we can determine that one method is better than another, referenced to theory. Evaluation of tools and methods with respect to theory is common in other engineering disciplines, as when a computational fluid dynamics model is critiqued because of its representation of eddies (Bui, 1999).

Our investigation reported in this paper is an example of assessing a design method with respect to established theories from political science and optimization. This method, the Pugh Method for Controlled Convergence, is of interest to our research agenda because it is commonly used in systems design. We are also investigating two other popular system design methods, Quality Function Deployment and the Analytic Hierarchy Process, and hope to report on these in future papers.

\subsection{Optimization, preferences, and design choice}

We make a basic assumption that a designer, studying two alternative designs, can order them by preference. He or she prefers Design A to Design B, prefers Design B to Design A, or is indifferent to the choice between $\mathrm{A}$ and $\mathrm{B}$. Furthermore, we assume that these preferences are transitive, so that any number of designs can be rank ordered by preference. Without these two assumptions, the idea of a better design or an improved design is lost, and there is no purpose left to engineering.

We further assume, along the lines of von Neumann (von Neumann and Morgenstern, 1947), that preference with respect to a set of alternative designs can be measured with cardinal, real numbers, such that higher numbers correspond to more preferred designs. This measure of preference is the theoretical ideal against which we compare the Pugh method.

Within this paper, we borrow from the Pugh method (and the other design-choice methods we plan to investigate), the limitation that preference for a design depends only on a vector of attributes used to describe each alternative. Thus, the ideal measure of preference for a design will take the form of a real function of the attributes of the design.

\section{THE PUGH METHOD FOR CONTROLLED CONVERGENCE}

The Pugh Method of Controlled Convergence is a common prescriptive approach to concept selection which evaluates a design by a vector of key attributes such as reliability, cost, weight, etc. The Pugh Method was developed by Stuart Pugh in response to his research conclusions regarding the shortcomings of the so-called "weight and rate method" of engineering decision making (Pugh 1991).

\subsection{The pugh procedure}

In the Pugh approach, each alternative solution is ranked relative to a "datum" configuration that is used as a benchmark [such as the market leader] (Pugh, 1991). Each attribute of each alternative is given a score of "+", "-", or "O" for "better than the datum", "worse than the datum" or "equivalent to the datum", respectively. The scores are determined by a group of subject matter experts through deliberation and discussion. Often, if consensus is not reached about an attribute for an alternative, the rating of " 0 " is used. Some method of elimination of alternatives is established to cull the set of alternatives. New alternative solutions are devised, often involving hybrid solutions between alternatives, and added to the set of alternatives and scored. The procedure is iterated until the team converges to a single preferred solution. The steps to Pugh are:

1. Gather set of initial concepts

2. Select evaluation criteria

3. Select one strong concept as the datum

4. SMEs rank each criteria for every concept against datum as better (+), worse (-), same (0)

5. Tally scores

6. Remove weak concepts

7. Generate new concepts

8. Select new datum (typically strongest concept)

9. Iterate until single concept has emerged as best 


\subsection{Pugh example}

Consider a problem where we are trying to select among a set of alternative automotive designs. Our criteria are fuel economy, horsepower, safety and passenger capacity. We are considering the following four alternatives: (1) Dodge Dart, (2) Corvette, (3) Jeep and (4) Caravan. The Dart is our current car, so we make it the datum and compare the alternatives to the Dart. The matrix is shown in Figure 1.

\begin{tabular}{|l|c|c|c|c|}
\cline { 3 - 5 } \multicolumn{1}{c|}{} & Dodge Dart & Corvette & Jeep & Caravan \\
\hline Fuel Economy & D & - & - & 0 \\
\hline Horsepower & A & + & + & 0 \\
\hline Safety & T & - & - & + \\
\cline { 1 - 4 } Passenger Capacity & U & - & + & + \\
\cline { 1 - 4 } & M & - & + &
\end{tabular}

Figure 1. Automobile selection Pugh Matrix 1

Based on this evaluation, the Caravan appears to be the superior choice due to its improved safety and passenger capacity compared to the Dart. Having evaluated a sports car, a sport utility vehicle and minivan, we learned that the minivan has better performance relative to our criteria compared to our datum vehicle. We also learned that the sports car is clearly inferior. Looking at alternatives similar to the minivan, we generate two new vehicle alternatives that we think would be competitive with the Caravan - we include the Outback and the Tahoe. The Outback is a new concept that is essentially a new class of automobile. The Tahoe is sort of a hybrid between the Jeep and the Minivan. We remove the Corvette from the alternatives. Since the Caravan was superior in the last matrix, we make the Caravan the new datum, create the new matrix and evaluate the alternatives. This is shown in Figure 2.

\begin{tabular}{|c|c|c|c|c|}
\hline & Caravan & Outback & Tahoe & Jeep \\
\hline Fuel Economy & \multirow{4}{*}{$\begin{array}{l}\text { D } \\
\text { A } \\
T \\
U \\
M\end{array}$} & + & - & - \\
\hline Horsepower & & + & + & + \\
\hline Safety & & + & + & - \\
\hline Passenger Capacity & & - & 0 & - \\
\hline
\end{tabular}

Figure 2. Automobile selection Pugh Matrix 2

In this iteration, the Outback is superior to the Datum based on our criteria. The Jeep is clearly inferior. Based on this information, the Jeep might be eliminated from further evaluation and other alternatives added. The process is repeated until we've converged on a single concept that is superior.

\subsection{Assumptions behind the Pugh Method}

The Pugh Method is one of several concept selection methods that evaluate each design alternative as a vector of attributes and aggregate the attribute evaluations into a choice (Pugh, 1991). Other such methods are Quality Function Deployment (Clausing, 1994) and the Analytical Hierarchy Process (Saaty, 2008). The Pugh Method ranks each attribute of each alternative as "better than", "worse than" or "indistinguishable from" the corresponding attribute of the datum design alternative. That is, each attribute of each design alternative is ordinally ranked against the corresponding attribute of the datum. (Ordinal refers to numbers such as first, second, tenth. This contrasts with cardinal numbers, such as 1, 7 or 9. Arithmetic requires cardinal numbers, it does not work with ordinal numbers.) Therefore, each comparison is done in a pairwise fashion against the datum. The primary assumptions made when using the Pugh Method are outlined in the following sections.

\subsubsection{Complete ordinal ranking}

In order to ordinally rank the attributes of each design alternative against the attributes of the datum, the ranking for each attribute must be complete. This means that, for every pair of design alternatives, $A$ and $\mathrm{B}$, each attribute can be described as "A is better than B", "B is better than A" or "neither A or B is better". Completeness is necessary for the Pugh Method per the procedure in 1.1. 


\subsubsection{Transitive ordinal ranking}

The ordinal rankings of the attributes of each design alternative must also be transitive. Transitivity implies that, for any three designs alternatives $\mathrm{A}, \mathrm{B}$ and $\mathrm{C}$, "A is better than $\mathrm{B}$ " and " $\mathrm{B}$ is better than $\mathrm{C}$ " for a given attribute, then "A is better than $C$ " for that same attribute. In the example in 1.2, if the Jeep has superior passenger capacity than the Dodge Dart and the Dodge Dart has superior passenger capacity to the Corvette, then Jeep has superior passenger capacity than the Corvette.

\subsubsection{Unrestricted ordinal ranking}

The Pugh Method, as well as any other ordinal design method, does not restrict the attribute rankings of the design alternatives. That is, any design alternative could be better or worse than any other design alternative relative to any particular attribute.

\subsubsection{Deterministic attribute values}

The Pugh Method assumes that the relative attribute values between the design alternatives is deterministic. In fact, all attribute values are predictions in the design process because the design has not yet been completed. Because the Pugh Method does not have a mechanism to handle the uncertainty associated with prediction or estimation, it assumes deterministic attributes or, at least, that the variability in the attribute values is negligible relative to the concept selection.

\subsubsection{Equally weighted attributes}

When tallying the attribute score for each design alternative, each attribute is weighted equally. An attribute that is better than the datum gets one added to its tally. An attribute worse than the datum gets a one subtracted from the tally. Therefore, all attributes have the same ability to impact the final tally and therefore, the final recommendation made by the matrix. In the above example, Safety, Fuel Economy and Horsepower are equally important to the design choice.

\subsubsection{Linear attributes}

The contribution of each attribute to the overall system utility is assumed to be linearly related to the attribute value. An improvement over the datum contributes one unit to the overall tally. Moreover, all improvements over the datum are assumed to be equally related to system value. A ten percent improvement in horsepower over the datum results in incrementing the tally by one unit, as does a $200 \%$ improvement in horsepower. Therefore, the differences in attributes values between design alternatives must be relatively "small," that is, not having a substantially different impact to overall system value.

\subsubsection{Preferential independence of attributes}

The Pugh method assumes that the attributes are preferentially independent. Preferential independence means that, improvement or degradation in one attribute for a particular design alternative does not imply an improvement or degradation in another attribute value for that design alternative, and does not impact the contribution of any other attributes to the overall system value (Thurston, 2006). An example of preferentially dependent attributes might be Mean Time Between Failures (MTBF) and Mean Time to Repair (MTTR). If the MTBF is extremely large, then the MTTR is less important. Conversely, if the MTTR is extremely low, then the MTBF is less important. The Pugh Method has no mechanism to deal with preferential interactions among attributes. Preferential independence is a consequence of linearity, but is significant enough that it bears pointing out separately.

\section{SOCIAL CHOICE THEORY}

Social Choice theory is concerned with making a decision involving a group of individuals. Each individual in the group has preferences. These individual preferences must somehow be aggregated into a preference ordering used by the group. The most familiar instance of Social Choice Theory is voting in elections. Each individual in the group provides a statement of preference--a vote--and these votes are aggregated to make a selection that represents the preference of the group. Social Choice Theory has been studied for decades and has a substantial body of associated research. 


\subsection{Generalization of social choice}

Social Choice Theory has been shown to apply to a much broader set of problems than originally envisioned. Don Saari has demonstrated that the application to Social Choice is a special case of the "Reductionist Approach," a class of problems that involves the following steps:

- Decomposition of a system into parts

- Analysis of the individual parts

- Aggregation of the analysis results to make inferences about the system

In the context of Social Choice, the society is decomposed into individuals. Each individual's preferences are analyzed and the analysis results are aggregated using a Social Choice Function in order to select the winner of the election. The Social Choice Function is intended to reflect the preferences of the Society. One such aggregation approach is to sum each individual's most preferred choice and select the candidate that is the preferred choice for the most voters--the so-called Plurality Vote (Saari, 2001). However, there are other methods such as Borda Count (de Borda, 1784) and Condorcet (1785). The Social Choice problem is graphically depicted in Figure 3.

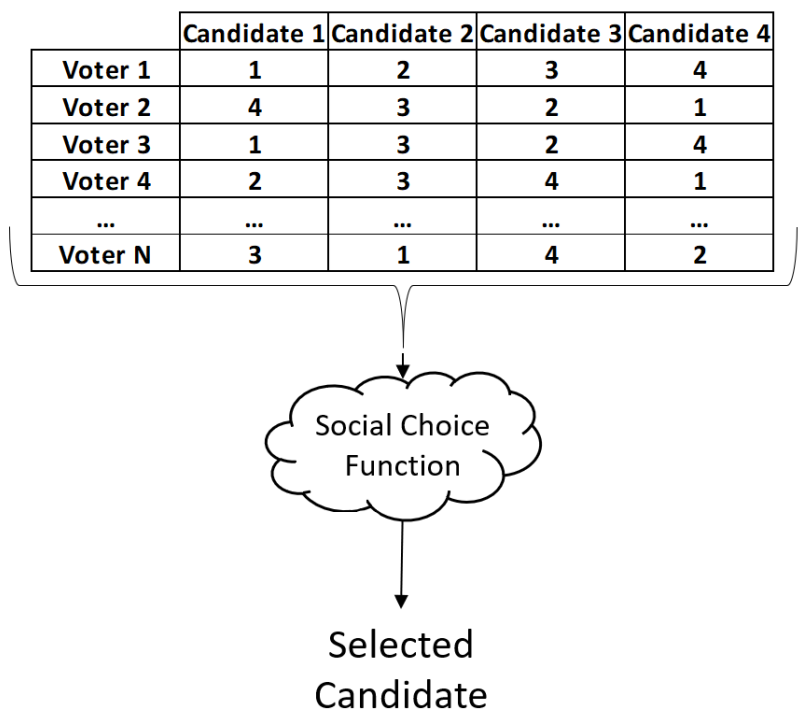

Figure 3. Graphical depiction of social choice problem

In this problem, each voter has preferences. The preferences are an ordinal ranking of candidates. For example, Voter 1 in Figure 3 has the following ordinal preference ranking:

Candidate $1>$ Candidate $2>$ Candidate $3>$ Candidate 4

The ">" symbol is read as "is preferred to." These preferences are analyzed in some way and aggregated using some Social Choice Function (Plurality, Borda, Condorcet, etc.) into a Social Choice intended to reflect the "will of the voters". However, this problem is more generic than voting in an election. Social Choice Theory has been shown to apply to nonparametric statistical hypothesis testing (Haunsperger and Saari 1992) and engineering design (Saari 2010, Hazelrigg 1996).

\subsection{Application to engineering design}

Recently, the mathematics and theory of Social Choice has been applied to engineering design (Saari 2010, Saari and Seiberg 2001, Franssen 2005, Hazelrigg 1996, etc.). Much of Social Choice Theory involves the aggregation of the complete, transitive and ordinal preference rankings of individuals into a complete, transitive and ordinal preference ranking of the society. This aggregation has been shown to lead to inconsistencies that result in an inferior choice to be selected by the group (Arrow 1951, Sen 1970). Franssen (2005) showed that the Pugh Matrix is analogous to a Social Choice problem where the attributes act as voters and the design alternatives act as candidates.

\subsection{Assumptions behind social choice theory}

Much of Social Choice Theory involves attempting to capture the "Will of the People" through aggregating their preferences using a Social Choice Function. The desired outcome is a result that is 
considered "fair". To this end, the Social Choice Theory relevant to this discussion requires that the preference profiles of each individual have the following attributes (Arrow, 1951):

- Complete Preferences

- $\quad$ Transitive Preferences

- $\quad$ Unrestricted Preferences

In Social Choice Theory, the preferences of each individual must be complete and transitive. That is, for any candidate $\mathrm{A}$ and $\mathrm{B}$, either the individual prefers $\mathrm{A}$ to $\mathrm{B}$, prefers $\mathrm{B}$ to $\mathrm{A}$ or is indifferent between $\mathrm{A}$ and $\mathrm{B}$. These preferences must also be transitive, meaning that if the individual prefers $\mathrm{A}$ to $\mathrm{B}$ and prefers B to C, then the individual must also prefer A to C. These axioms used in Social Choice Theory are equivalent to the assumptions made by the Pugh Method. In Social Choice Theory, the preferences of the individual must be unrestricted. This means that the preference ordering of the candidates cannot be limited by the Social Choice Function. The Social Choice Function cannot reject the preferences of individuals who prefer one candidate to another. Similarly, engineering concept selection methods cannot assume certain attribute performance profiles cannot exist or remove attributes from selection method that have certain design rankings.

The assumptions made by the Pugh Method encompass the assumptions made by Social Choice Theory regarding the preference profiles of individuals. Therefore, the inconsistencies described in the following sections are relevant to both the Social Choice problem as well as the Pugh Method. One might notice that there are more assumption made by the Pugh Method than Social Choice Theory. These additional assumptions made by the Pugh Method are the assumptions about the attributes and design alternatives that are required to correctly transform cardinal attributes into ordinal attribute performance profiles that can be evaluated independently. Once the attributes have been reduced to ordinal profiles, the mathematics are equivalent between Social Choice Theory and the Pugh Method. If any of the assumptions is rejected, then the mathematics of Social Choice Theory no longer apply. We have explored the impact of relaxing the assumption Equally Weighted Attributes, and will report this in a later paper. The remaining assumptions of the Pugh Method are sufficient to subject it to the inconsistencies and paradoxes identified in Social Choice Theory.

\subsection{Relevant inconsistencies in social choice}

In Social Choice Theory, paradoxes and inconsistencies have been identified and proven in the literature (Arrow, 1951, Sen 1970, Condorcet, 1785, Saari 2001). Many of the paradoxes involve aggregation of the individual preferences into a result considered "unfair." For example, if all individuals unanimously agree that A is preferred to B, it would be unfair for the Social Choice Function to select B. Social Choice Functions which guarantee that this unfair outcome will not occur are called Pareto Efficient (Pareto 1927). Various Social Choice Functions can violate Pareto Efficiency (Arrow 1951).

\subsubsection{Transitive societal preferences}

Social Choice Theory requires that the preferences of each individual are transitive. If an individual does not have transitive preferences, they are described as "irrational" and are subject to the "Money Pump" argument (Peterson 2017). The Money Pump argument uses an individual with intransitive preferences-say she prefers Coke to Pepsi, prefers RC Cola to Coke and prefers Pepsi to RC Cola. An opportunistic individual could exploit this preference ordering by using the following procedure:

1. Give the irrational individual a Pepsi

2. Offer to sell her a Coke for $1 \varnothing$ (she will since she prefers Coke to Pepsi)

3. Offer to sell her RC Cola for $1 \varnothing$ (she will since she prefers RC Cola to Coke)

4. Offer to sell her Pepsi for $1 \varnothing$ (she will since she prefers Pepsi to RC Cola)

5. Return to Step 2 or until she has no more money

Similarly, it is desirable for the preference ranking generated by the Social Choice Function to be transitive. If society preferred Bush to Nader, preferred Nader to Gore and preferred Gore to Bush, it is impossible to select the presidential candidate that society prefers because, no matter who is selected, there is a candidate society prefers more-no best candidate exists under intransitive societal preferences. 


\subsubsection{Condorcet paradox}

The Condorcet voting method involves performing a pairwise comparison between each pair of candidates and aggregating the pairwise results into a candidate selection. The Condorcet Winner is defined as the candidate who is preferred by a majority of voters to every other candidate in a pairwise vote. For example, consider the three voter profiles shown below:

- $\quad$ Voter 1: Candidate $1>$ Candidate $2>$ Candidate 3

- $\quad$ Voter 2: Candidate $2>$ Candidate $1>$ Candidate 3

- Voter 3: Candidate $1>$ Candidate $3>$ Candidate 2

Performing the Condorcet method shows that the majority of voters (2/3) prefer Candidate 1 to Candidate 2 and a majority of voters (all of them) prefer Candidate 1 to Candidate 3 . Therefore, the Condorcet winner would be Candidate 1. However, consider the three voter profiles below:

- $\quad$ Voter 1: Candidate $1>$ Candidate $2>$ Candidate 3

- $\quad$ Voter 2: Candidate $3>$ Candidate $1>$ Candidate 2

- Voter 3: Candidate $2>$ Candidate $3>$ Candidate 1

Using this profile, a majority of voters (2/3) prefer Candidate 1 to Candidate 2, a majority (2/3) of voters prefer Candidate 2 to Candidate 3 and a majority of voters (2/3) prefer Candidate 3 to Candidate 1 . A Condorcet Winner does not exist. Moreover, a winner does not exist because the resulting preferences of the group are intransitive. Clearly, this Social Choice Function does not guarantee that the resulting Societal Preference Profile is transitive. Saari and Sieberg (2001) show that this outcome occurs alarmingly often when performing a decision procedure involving paired comparisons.

\section{THEORETICAL EVALUATION OF PUGH METHOD}

We have evaluated the Pugh Method in a two-step process. In the first step, reported here, we assume that all the assumptions made by the Pugh Method are valid for engineering design. That is, the concept selection problem can be cast as an ordinal ranking of attributes performance profiles that are complete and transitive. In this context, the Pugh Method is subject to the paradoxical outcomes of Social Choice Theory. The second evaluation step, in which Pugh will be compared to a direct measure of preference as a function of attributes, cannot be reported in the space here, but will be shown in a later paper.

Making all of the assumptions made by the Pugh Method, we will analyze the potential paradoxical outcomes generated by aggregating the ordinal performance profiles using the Pugh Method. The Pugh Method is a pairwise aggregation procedure because the attributes of each design alternative are compared to the datum and aggregated. Therefore, the Condorcet Paradox is expected to apply.

\subsection{Transitive design alternative ordering}

Similar to the Social Choice requirement for Transitive Societal preferences, engineering Concept Selection methods require transitive design alternative rankings. As in the Social Choice context, a best candidate cannot be selected from intransitive rankings because a best candidate does not exist.

\subsection{Condorcet triples in pugh method}

Consider the three microwave designs and three attributes shown in Figure 4.

In this example, more power is preferred, lower cost is preferred, and more volume is preferred. In order to evaluate these designs using the Pugh Method, a datum must be selected. Selecting Design A as the datum and evaluating the matrix yields the Pugh Matrix shown in Figure 5 with the aggregated scores included at the bottom of the matrix.

\begin{tabular}{|l|c|c|c|}
\cline { 2 - 4 } \multicolumn{1}{c|}{} & Design A & Design B & Design C \\
\hline Power $(\mathrm{W})$ & 1100 & 1200 & 1000 \\
\hline Cost $(\$)$ & 121.53 & 139.99 & 2.99 \\
\hline Volume $\left(\mathrm{ft}^{3}\right)$ & 1.4 & 1.2 & 1.1 \\
\hline
\end{tabular}

Figure 4. Microwave design selection problem 


\begin{tabular}{|l|c|c|c|}
\hline & Design A & Design B & Design C \\
\hline Power $(\mathrm{W})$ & \multirow{4}{*}{ DATUM } & + & - \\
\cline { 1 - 1 } Cost $(\$)$ & - & - \\
\cline { 1 - 3 } Volume $\left(\mathrm{ft}^{3}\right)$ & & + & + \\
\cline { 3 - 4 } & & 1 & -1 \\
\hline
\end{tabular}

Figure 5. Microwave selection using Pugh, $A$ as datum

Using the Pugh Method, Design B would emerge as the best design alternative. However, selecting Design B as the datum yields the matrix shown in Figure 6.

\begin{tabular}{|l|c|c|c|}
\hline & Design A & Design B & Design C \\
\hline Power $(\mathrm{W})$ & - & & - \\
\hline Cost $(\$)$ & + & \multirow{2}{*}{ DATUM } & + \\
\cline { 1 - 1 } Volume $\left(\mathrm{ft}^{3}\right)$ & - & & + \\
\cline { 1 - 1 } & -1 & & 1 \\
& & &
\end{tabular}

Figure 6. Microwave selection using Pugh, $B$ as datum

With Design B as the datum, Design C emerges as the preferred design alternative. Finally, selecting Design $\mathrm{C}$ as the datum yields the matrix shown in Figure 7.

\begin{tabular}{|l|c|c|c|}
\hline & Design A & Design B & Design C \\
\hline Power $(\mathrm{W})$ & + & + & \\
\cline { 1 - 3 } Cost $(\$)$ & + & - & \multirow{2}{*}{ DATUM } \\
\cline { 1 - 3 } Volume $\left(\mathrm{ft}^{3}\right)$ & - & - & \\
\cline { 1 - 3 } & 1 & -1 & \\
\cline { 2 - 3 } & & &
\end{tabular}

Figure 7. Microwave selection using Pugh, $C$ as datum

With Design $\mathrm{C}$ as the datum, Design A emerges as the best design. The selected design is purely a function of which design alternative was selected as the datum. No matter which design is selected, a better design can be found by selecting a different datum. This is an intransitive outcome and is a function of the Condorcet Paradox. The attribute performance profiles are shown below:

- Power: Design B > Design A > Design C

- Cost: Design A > Design C > Design B

- Volume: Design C > Design B > Design A

\subsection{Other Pugh Method inconsistencies}

Saari and Seiberg (2001) conclude that the Condorcet Paradox is a result of "lost information" from only looking at each pair in isolation. Though the Pugh Method is subject to the Condorcet Paradox, it has additional paradoxical outcomes due to not evaluating all the pairs. The Pugh Method only evaluates each design alternative to the datum--it does not compare the design alternatives to each other. In the Microwave example above, when Design A is the datum, Design A is compared to Design B and Design A is compared to design C. Design B and Design C are not compared. This leads to the differences between Design B and Design C being neglected if they are both better than the datum or both worse that the datum. Consider the microwave design problem with the following preference profile:

- Power: Design A > Design B > Design C

- $\quad$ Cost: Design A > Design B > Design C

- Volume: Design B > Design C > Design A 
Design A is the Condorcet Winner in this example. Design A is better than Design B and Design C for both Power and Cost. However, when Design $\mathrm{C}$ is the datum, the differences between Design A and Design B are neglected for Cost and Power. The Pugh Matrix for this problem is shown in Figure 8.

\begin{tabular}{|c|c|c|c|}
\hline & Design A & Design B & Design C \\
\hline Power $(\mathrm{W})$ & + & + & \multirow{2}{*}{ DATUM } \\
\hline Cost $(\$)$ & + & + & \multirow{2}{*}{ Volume $\left(\mathrm{ft}^{3}\right)$} \\
\cline { 1 - 3 } & - & + & \\
\cline { 1 - 3 } & 2 & 3 & \\
\cline { 2 - 3 } & & &
\end{tabular}

Figure 8. Pugh Method paradoxical outcome

Despite Design A being the Condorcet Winner in this example, the Pugh Method recommends Design B as the best design alternative.

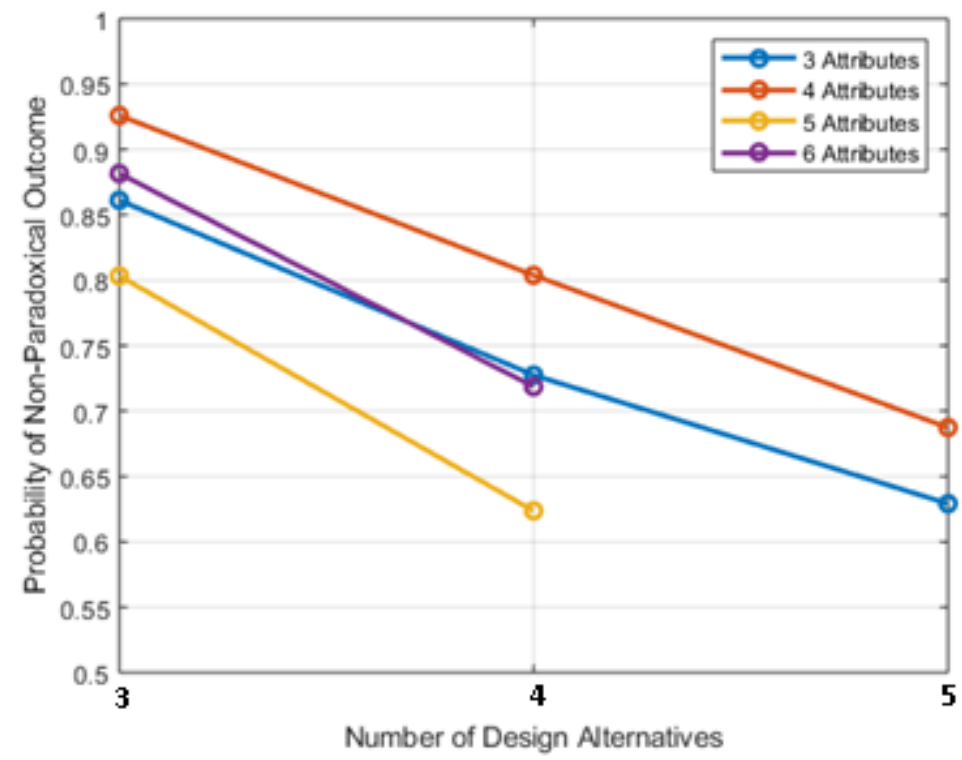

Figure 9. Probability of correct ordering of alternatives in Pugh Method

\subsection{Frequency of occurrence}

To determine how frequently paradoxical outcomes occur in the Pugh Method, we performed the Pugh Method procedure on every permutation of preference orderings for various numbers of design alternative and attributes. This brute force approach quickly becomes unwieldy. As the number of design alternatives and attributes increases beyond five the number of permutations reaches the billions. The results of this analysis is shown in Figure 9.

Figure 9 shows, for example, that the frequency of a paradoxical outcome (an intransitive preference loop or an incorrect choice due to incomplete comparisons) from the Pugh Method is $38 \%$ for a concept selection problem of four alternatives and five attributes.

\section{CONCLUSION}

Debate between the Pugh method and decision-based design has focused on creativity and iteration (Frey et al., 2009). Here we concentrate simply on the step in which Pugh ranks design alternatives. The +, -, 0 paired comparisons which Pugh regards as a virtue of simplicity, we find as an unnecessary sacrifice of information that may be essential to a rational choice. Pugh lacks the ability to express degrees of difference in attributes, nonlinearity of attributes, uncertainty or preference-dependent interaction of attributes, all of which may be known to designers, but which they must put aside to use Pugh. We seek instead an even simpler evaluation in which designers bring what they know about each alternative along with their preferences, and express that information and those preferences with basic mathematics. Good designers enjoy and thrive on math, they do not hide from numbers. 


\section{ACKNOWLEDGEMENT}

This work was supported in part by the National Science Foundation under grant CMMI-1663109.

\section{REFERENCES}

Arrow, K. (1951), Social Choice and Individual Values, John Wiley and Sons Inc., New York, NY.

Bui, T. (1999), "A parallel, finite-volume algorithm for large-eddy simulation of turbulent flows", NASA Technical Memorandum 1999-206570, NASA Armstrong Flight Research Center, Edwards, CA.

Clausing, D. (1994), Total Quality Development: A Step-By-Step Guide to World-Class Concurrent Engineering, ASME Press, New York, NY.

Collopy P. and Mesmer B. (2015), "Report on the science of systems engineering workshop", AIAA Paper 20151865. American Institute of Aeronautics and Astronautics, Reston, VA.

Condorcet, J. (1785), An Essay on the Application of Analysis to the Probability of Decisions Rendered by A Plurality of Votes, Reprinted by Chelsea Publishing, New York, NY.

de Borda, M. (1784), "On elections by ballot", Histoire de l'Academie Royale des Sciences, pp. 31-34.

Franssen, M. (2005), "Arrow's theorem, multi-criteria decision problems and multi-attribute preferences in engineering design", Res. in Eng. Des., Vol. 16, pp. 42-56.

Frey D, Herder P., Wijnia Y., Subrahmanian E., Katsikopoulos K. and Clausing D. (Mar. 2009), "The pugh controlled convergence method: Model-based evaluation and implications for design theory", Research in Engineering Design, Vol. 20, pp. 41-58.

Haunsperger, D. and Saari, D. (1992), "Dictionaries of paradoxes for statistical tests on k-samples", Jour. Amer. Statistical Assoc., Vol. 87, pp. 249-272.

Hazelrigg, G. (1996), “The implications of arrow's impossibility theorem on approaches to optimal engineering design", ASME J. Mech Des., Vol. 118, pp. 161-164.

Pareto, V. (1927), Manual of Political Economy, Augustus M. Kelley Publishing, New York, NY.

Peterson, M. (2017), An Introduction to Decision Theory, Cambridge University Press, New York, NY.

Pugh, S. (1991), Total Design: Integrated Methods for Successful Product Engineering, Addison Wesley Publishing Company, Reading, MA.

Saari, D. (2001), Decisions and Election: Explaining then Unexplained, Cambridge University Press, New York, NY.

Saari, D. (2010), "Aggregation and multilevel design for systems: Finding guidelines", ASME J. Mech Des., Vol. 132.

Saari, D. and Seiberg, K. (2001), “Are pairwise comparisons reliable?”, Res. Eng. Des., Vol. 15, pp. 62-71.

Saaty, T. (2008), Group Decision Making: Drawing Out and Reconciling Differences, RWS Publications, Pittsburgh, PA.

Sen, A. (1970), "The impossibility of the paretian liberal", Journal of Political Economy, Vol. 78, pp. 152-157.

Thurston, D. (2006), "Multi-attribute Utility Analysis of Conflicting Preferences", In: Lewis, K.E. et al. (Ed.), Decision Making in Engineering Design, ASME Press, New York, New York. pp. 125-133.

von Neumann, J. and Morgenstern, O. (1947), Theory of Games and Economic Behavior, Princeton University Press, Princeton, pp. 16-31. 